\title{
Reservoir computing based on quenched chaos
}

\author{
Jaesung Choi, Pilwon Kim* \\ Department of Mathematical Sciences, Ulsan National Institute of Science and Technology(UNIST), Ulsan Metropolitan City 44919, Republic of Korea
}

\section{A R T I C L E I N F O}

\section{Article history:}

Received 27 August 2019

Revised 3 February 2020

Accepted 14 July 2020

Available online 23 July 2020

\section{Keywords:}

Reservoir computing

Quenched chaos

Chaos computing

\begin{abstract}
A B S T R A C T
Reservoir computing (RC) is a brain-inspired computing framework that employs a transient dynamical system whose reaction to an input signal is transformed to a target output. One of the central problems in $\mathrm{RC}$ is to find a reliable reservoir with a large criticality, since computing performance of a reservoir is maximized near the phase transition. In this work, we propose a continuous reservoir that utilizes transient dynamics of coupled chaotic oscillators in a critical regime where sudden amplitude death occurs. This "explosive death" not only brings the system a large criticality which provides a variety of orbits for computing, but also stabilizes them which otherwise diverge soon in chaotic units. The proposed framework shows better results in tasks for signal reconstructions than RC based on explosive synchronization of regular phase oscillators. We also show that the information capacity of the reservoirs can be used as a predictive measure for computational capability of a reservoir at a critical point.
\end{abstract}

(c) 2020 Elsevier Ltd. All rights reserved.

\section{Introduction}

Recently, reservoir computing has emerged as a promising computational framework for utilizing a dynamical system for computation. While an input stream perturbs the transient intrinsic dynamics of a medium("reservoir"), a readout layer is trained to extract features out of such perturbations to approximate a target output. Due to its complex high-dimensional dynamics, the reservoir serves as a vast repertoire of nonlinear transformations that can be exploited by the readout. The major advantage of reservoir computing is their simplicity in training process compared to other neural networks. Another advantage is their universality in that they can be realized using physical systems, substrates, and devices [12,14,34].

There is the hypothesis that a system can exhibit maximal computational power at a phase transition between ordered and chaotic behavioral regimes $[18,19]$. It has been observed that the brain operates near a critical state in order to adapt to a great variety of inputs and maximize information capacity [3-5]. Perturbations occurring in a critical regime neither spread nor die out too quickly, providing the most flexibility to the system $[10,16]$. This concept of "computation at the edge of chaos" may also have an implication to material computation, whereby a material has the most exploitable properties [27]. More extensive review on this subject can be found in Munoz [28].

\footnotetext{
* Corresponding author.

E-mail address: pwkim@unist.ac.kr (P. Kim).
}

In RC, designing a reservoir which has a large criticality is important to perform complex tasks. In case of a reservoir based on continuous dynamical systems, one can create criticality by tuning intrinsic parameters so that the reservoir operates at a bifurcation point across which the dimension of the attractor abruptly declines. We call such system a critical reservoir. A system of coupled oscillator exhibits a first order transition from incoherent state to synchronized state that occurs under a specific relation between the coupling strength and connectivity, which is called explosive synchronization. In the previous work [7], we showed that a reservoir of coupled Kuramoto oscillators near explosive synchronization forms a critical reservoir and performs excellent computations.

Amplitude death(AD) is another way to create a criticality in coupled oscillatory units. It indicates complete cessation of oscillations induced from change in intrinsic parameters of the system. The occurrence of $\mathrm{AD}$ has been found in the case of chemical reactions [8,11], neuronal systems [13,29] and coupled laser systems $[15,37]$. It has been also reported that AD can occur abruptly in a system of coupled nonlinear oscillators [21,35,36,39]. Such simultaneous cessation of oscillations is the first order transition to $\mathrm{AD}$ and called "explosive death"(ED).

In this work, we focus on computing ability of chaotic systems near a criticality created in the form of ED. There have been many researches on chaos computing [2,22], even in the context of RC [17,23-25]; Chaos computing takes advantage of an infinite number of orbits/patterns inherent in the attractor to be used for particular computational tasks. It also utilizes the sensitivity to initial conditions of chaotic systems to perform rapid switching between 


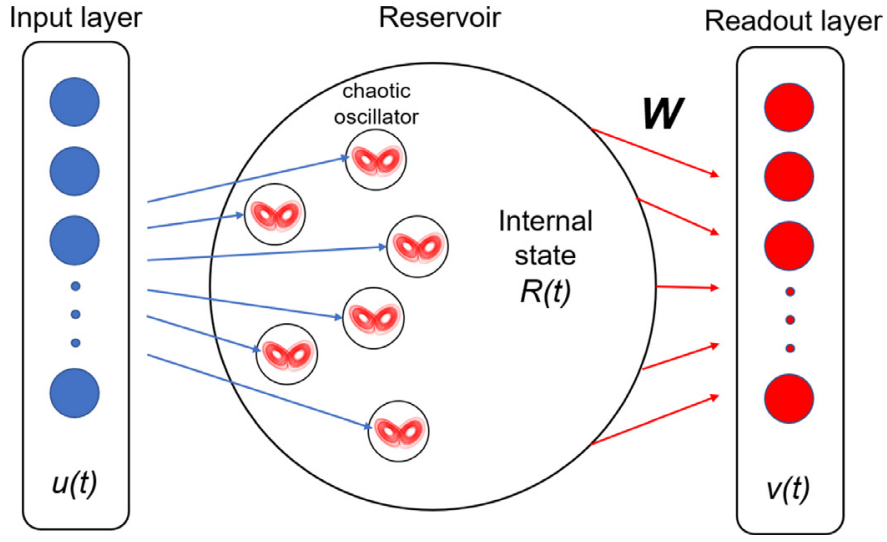

Fig. 1. Schematic of RC of this work. The nodes in the reservoir are chaotic oscillators and their internal dynamics is aroused by the input signal $u(t)$. Only the weight matrix $\mathbf{W}$ connecting the reservoir state $R(t)$ needs to be trained to generate a desired output $v(t)$.

computational modes. However, chaos computing often has a control problem to stabilize particular orbit.

Our major goal is to construct a chaos based reservoir with a large criticality induced from explosive death. We focus on how to create an easily-adjustable criticality in reservoirs, since, as we will see in Section 4, a criticality provides a reliable criterion to choose the parameter values for maximized computing performance. We use the coupled chaotic oscillators and adjust a coupling strength so that they remain near the stage of ED. A reservoir in such critical regime provides a large variety of orbits in transient dynamics which can be used in computational tasks. Different from previous chaotic computing methods, the reservoirs still remain in a regular regime during computation, but close enough to chaos to enjoy its richness. We also investigate how such quenched chaos enhances the criticality in RC. To show the contribution of chaos in criticality, we compare the computation performance of a chaotic critical reservoir with a non-chaotic critical reservoir.

\section{Model}

\subsection{Setup for reservoir computing}

The scheme for RC of chaotic oscillators is illustrated in Fig. 1.

The setup is divided into three parts: an input layer, a reservoir, and a readout layer. The reservoir in the middle consists of interconnected nodes of chaotic oscillators, some of which are also connected to the input signal. The nodes in the reservoir evolves dynamically in reaction to the temporal input signals $u(t)$ and their collective states in the reservoir form the reservoir state $R(t)$. The role of the reservoir can be viewed as a nonlinear transformation of the input $u(t)$ to a space represented by $R(t)$. The third part of RC is the readout layer where the training process is carried out. In the readout layer, a set of sampled reservoir states $R_{k}=R\left(t_{k}\right), k=1,2, \ldots$ are used to generate the final desired output $v(t)$ through a linear transformation as

$v(t)=\mathbf{W} \mathbf{R}$

where $\mathbf{R}$ is the reservoir state vector which consists of a temporal collection of $R_{k}$, and $\mathbf{W}$ is a weight matrix to be found in the training process. Since training the RC system only involves solving a linear Eq. (1) for the weight matrix $\mathbf{W}$, the training cost can be substantially reduced compared to conventional neural network approaches.

\subsection{Reservoir of nonidentical chaotic elements}

We consider a reservoir that consists of $N$ nodes of Lorenz systems coupled via a mean-field diffusion as,

$$
\begin{aligned}
\frac{1}{w_{i}} \frac{d x_{i}}{d t} & =10\left(y_{i}-x_{i}\right)+K\left(Q \bar{x}-x_{i}\right) \\
\frac{1}{w_{i}} \frac{d y_{i}}{d t} & =-x_{i} z_{i}+\rho x_{i}-y_{i} \\
\frac{1}{w_{i}} \frac{d z_{i}}{d t} & =x_{i} y_{i}-\frac{8}{3} z_{i}
\end{aligned}
$$

where $i=1, \ldots, N$ is the index of the oscillators and $\bar{x}=\frac{1}{N} \sum_{i=1}^{N} x_{i}$ is the mean field of the state variable $x$. The parameter $K$ is the strength of coupling and $0 \leq Q \leq 1$, is the intensity of the mean field. Here we use $Q=0.7$, following [35].

Each single node exactly coincides with the conventional Lorenz system if $K=0$ with $w_{i}=1$. If the frequencies of the nodes $w_{i}$ are identical, then the system in Eq. (2) undergoes ED upon a change of the parameters [35]. In the following sections, we confirm that ED persistently occurs in Eq. (2) with a suitable distribution of the frequency $w_{i}$. When running the system in Eq. (2) as a reservoir, we therefore set the parameters for the system to be posed in a critical regime where the phase transition occurs. The adjustment of the parameters according to an order parameter will be discussed in Section 3.

\subsection{Readout and training}

The chaotic reservoirs are applied to supervised tasks of which training data comes in the form of $(u, v)$ where $u(t)=\left(u^{1}(t), \ldots, u^{p}(t)\right) \in \mathbb{R}^{p}$ is an input signal and $v(t)=$ $\left(v^{1}(t), \ldots, v^{q}(t)\right) \in \mathbb{R}^{q}$ is a target output. We assign $p$ nodes of the reservoir as input nodes. Before the training process starts, we run the network until it reaches an amplitude death state. Then the input stream $u(t)$ is fed to the reservoir, in a way that the value of $x(t)$ in the input nodes are perturbed by adding $u(t)$. All evolutionary activities of the nodes are measured to compute the output function $f_{\text {out }}=\left(f_{\text {out }}^{1}, \ldots, f_{\text {out }}^{q}\right) \in \mathbb{R}^{q}$.

We construct a linear output function $f$ that maps the reservoir state $R(t)$ to a desired output $v(t)$ as in the Eq. (1). In the readout process, it is reasonable to use the past values of the nodes as well as the current ones, to exploit the rich dynamics of the chaotic reservoirs. Here we set the output function $f$ to take past $s$ sampled values of the frequency $x_{i}^{\prime}=\frac{d x_{i}}{d t}$ at discrete times $t-\Delta t, t-2 \Delta t, \ldots, t-s \Delta t$ as an internal state of the reservoir.

We define the output function $f_{\text {out }}=\left(f_{\text {out }}^{1}, \ldots, f_{\text {out }}^{q}\right) \in \mathbb{R}^{q}$ of $(s$, $\Delta t$ )-type as

$f_{\text {out }}^{l}(t)=\sum_{i=1}^{N} \sum_{j=1}^{s} w_{i, j}^{l} x_{i}^{\prime}(t-(j-1) \Delta t), \quad l=1, \ldots, q$

Note that Eq. (3) essentially implements the linear readout process of RC in Eq. (1). Here $w_{i, j}^{l}$ are weights to be determined from the training process for each computational task, so that $f_{\text {out }}(t)$ is as close to $v(t)$ as possible. For example, if the output data is a time series $v\left(t_{1}\right), v\left(t_{2}\right), \ldots, v\left(t_{M}\right)$, the mean-square error

$\frac{\sum_{i=1}^{M}\left\|v\left(t_{i}\right)-f_{\text {out }}\left(t_{i}\right)\right\|^{2}}{\sum_{i=1}^{M}\left\|v\left(t_{i}\right)\right\|^{2}}$

can be used to determine the weights $w_{i, j}^{l}$. Note that minimizing the error in Eq. (4) with respect to the weights $w_{i, j}^{l}$ in Eq. (3) corresponds to a linear least squares problem. 
(a)

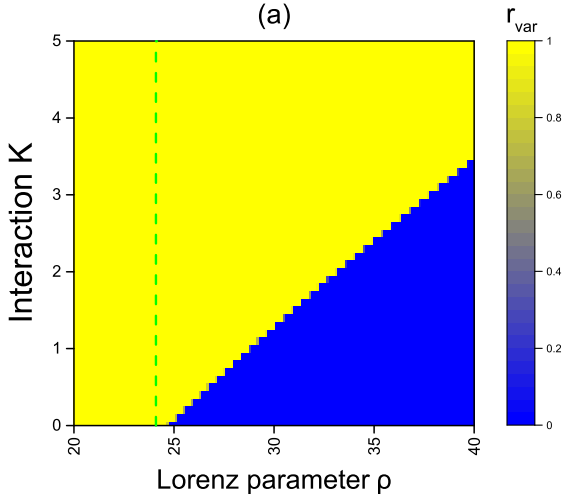

(b) $\rho=28$

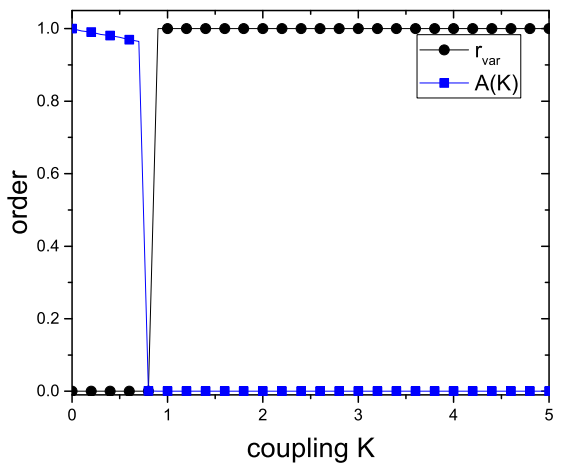

(c) $\mathrm{K}=2.2$

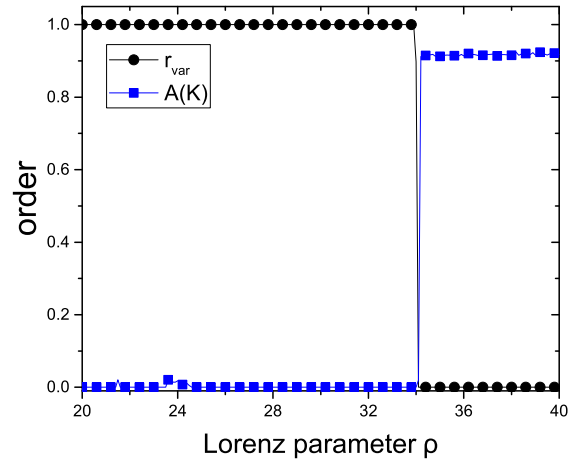

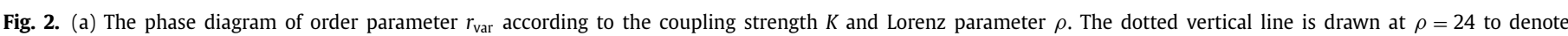

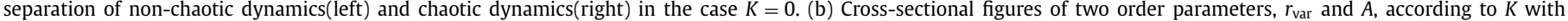
$\rho=28$ fixed. (c) Cross-sectional figures of two order parameters, $r_{\text {var }}$ and $A$, according to $\rho$ with $K=2.2$ fixed.

\section{Creating a criticality by the explosive death}

Benefit of using the coupled chaotic systems in Eq. (2) as a reservoir is that one can easily create a large criticality with a first order phase transition in the system. Across a critical point of the coupling force, the compound oscillatory motions of the system collapse into an equilibrium point. This fixed equilibrium state near a critical point is used as the ground state for reservoir computing, where the system always returns to after every computation, erasing unnecessary information from previous evaluations and preparing for the next inputs.

To look for a possible phase transition in Eq. (2), we define an order parameter $r_{\mathrm{var}}$ in terms of the variation of amplitudes, as

$r_{\mathrm{var}}=\frac{1}{N} \sum_{j=1}^{N} \exp \left(-c \operatorname{var}_{j}\right), \quad c>0$

where $\operatorname{var}_{j}$ is the temporal variance of the frequency $x_{j}^{\prime}(t)$ [7]. This is a measure for desynchrony that sensitively shows a degree of deviation of oscillators from a steady frequency. In the ground state, the temporal variance of the frequency should be kept low for reliable computations. Note that, for each oscillator, the temporal variance of the frequency becomes 0 if a strong coupling strength holds oscillators in a phase-locked state, keeping their common frequency steady. We also use another order parameter based on the normalized average amplitude as

$A(K)=\frac{a(K)}{a(0)}, \quad a(K)=\frac{\sum_{i=1}^{N}\left(\left\langle x_{i, \max }\right\rangle_{t}-\left\langle x_{i, \min }\right\rangle_{t}\right)}{N}$,

which is widely used for chaotic oscillators [30,31,35]. Note that $A(K)=0$ implies a complete cessation of oscillations and $A(K)=1$ implies nondepressed chaotic oscillations.

If the frequencies of nodes in Eq. (2) are identical, then the system exhibits explosive death, the discontinuous transition from the oscillatory state to the completely quenched state [35]. Indeed, Eq. (2) with nonidentical natural frequencies still exhibits the same phenomena. Fig. 2(a) is the phase diagram of the order parameter $r_{\text {var }}$ in the $\rho-K$ parameter plane. We used $N=100$ oscillators whose natural frequencies follow the uniform distribution in [1,1.3]. The dynamical states of system is obtained by backward continuation from a large value of $K$ [35]. The order parameter $r_{\mathrm{var}}$ was averaged between $t=[3900,4000]$. The diagonal line in Fig. 2(a) clearly splits the parameter plane according to the value of $r_{\mathrm{var}}$, indicating that the discontinuous phase transition occurs with respect to both $\rho$ and $K$. The graphs in Fig. 2(b) and (c) indicate cross-sectional figures of two order parameters in $K$ and $\rho$ directions, respectively. It is verified that both of the order param- eters $r_{\mathrm{var}}$ and $A$ exhibit extremely abrupt jump at the same critical point.

\section{Results}

\subsection{Numerical tests}

In the following numerical examples to test the learning ability of the oscillator networks, we use the $(10,0.1)$-type readout. That is, the output function $f_{\text {out }}$ at $t$ is obtained from 10 previous sampled values of the oscillator frequencies $x_{i}^{\prime}(t), \ldots, x_{i}^{\prime}(t-0.9)$ in Eq. (3).

We set up two types of tasks, inferring missing variables(Task 1 and Task 2) and filtering signals(Task 3), all of which require the presence of long-term memory for proper execution. In Task 1, RC is used to reconstruct evolutionary value of the variable $y(t)$ (or $z(t))$ of the Rössler system

$$
\begin{aligned}
& \frac{d x}{d t}=-y-z \\
& \frac{d y}{d t}=x+a y \\
& \frac{d z}{d t}=b+z(x-c)
\end{aligned}
$$

from observation of a single variable $x(t)$. That is, $u(t)=x(t)$ is the input signal and $v(t)=y(t)$ is the target output of RC in the task. We use $a=0.2, b=0.2$ and $c=5.7$ which are commonly used for study of chaotic behavior [20]. Similarly, Task 2 is to infer missing variables of the Chua's circuit

$$
\begin{aligned}
& \frac{d x}{d t}=\alpha(y-\phi(x)) \\
& \frac{d y}{d t}=x-y+z \\
& \frac{d z}{d t}=-\beta y
\end{aligned}
$$

where $\phi(x)=m_{1} x+1 / 2\left(m_{0}-m_{1}\right)(|x+1|-|x-1|)$. RC is trained to reconstruct a desired output $v(t)=y(t)$ from the input $u(t)=$ $x(t)$. The parameters are set as $\alpha=15.6, m_{0}=-8 / 7, m_{1}=-5 / 7$ and $\beta=28$ which lead to chaotic behavior.

Task 3 is to learn to generate the filtered scalar output

$v(t)=\frac{1}{m} \sum_{k=1}^{m}\left(a u(t-k)+b u(t-k)^{2}+c u(t-k)^{3}\right)$

which is determined from the past $m$ values of an input stream $u(t)$. Here $a, b$ and $c$ are some nonzero parameters. If $m=1$, the 
(a) Task 1

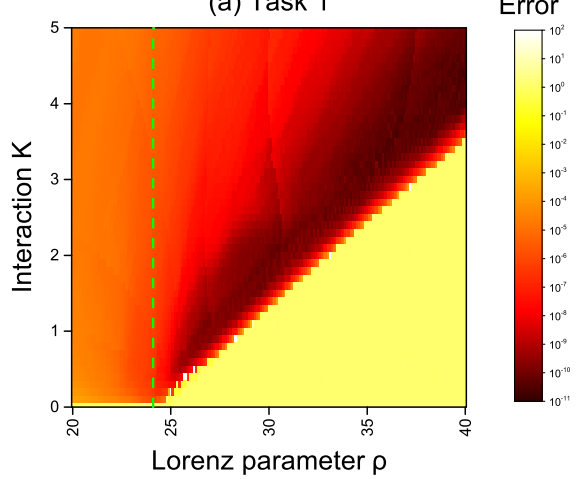

(b) Task 2

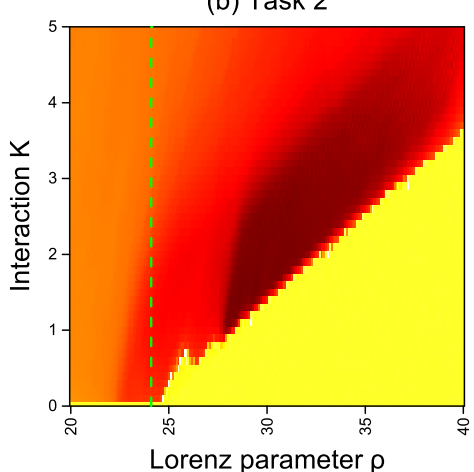

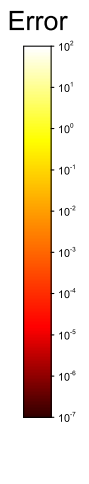

(c) Task 3

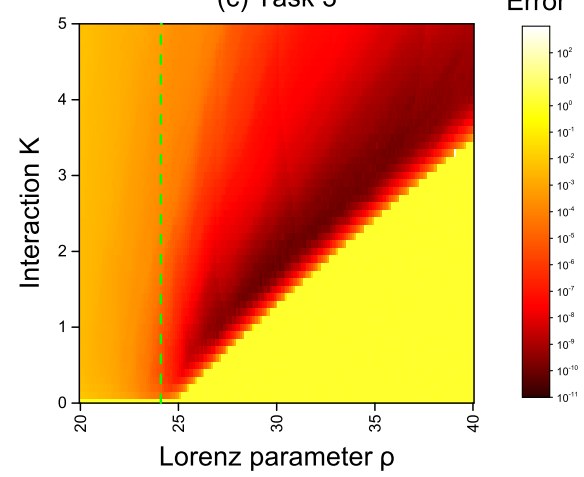

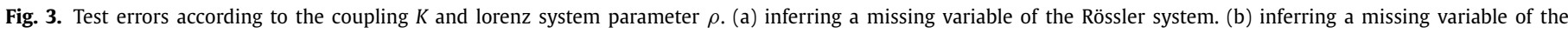
Chua's circuit. (c) filtering the Mackey-Glass equation.

task is simply to implement a polynomial function of the current value of the input. The task becomes more challenging as $m$ increases, requiring long-term memory to evaluate averaged values. In our tasks, we use the parameters $m=20, a=1, b=3$ and $c=5$, and have the input $u(t)$ generated from the Mackey-Glass equation

$$
\begin{aligned}
& \frac{d x}{d t}=\beta \frac{x(t-\tau)}{1+x(t-\tau)^{n}}-\gamma x, \quad \beta=0.2, \gamma=0.1 \\
& \tau=17 \text { and } n=10 .
\end{aligned}
$$

which provides standard benchmark task for chaotic series handling [6].

In each task, the continuous input signal $u(t)$ and the target signal $v(t)$ are generated for $t \in[0,6000]$. To make sure that the system is positioned in the reliable ground state, we skip first 1000 time steps of the output. The training process is applied to match $f_{\text {out }}$ to $v(t)$ over the 4000 discrete time steps, $t=1001,1002, \ldots, 5000$. That is, the readout weights $w_{i, j}^{l}$ in Eq. (3) are determined to minimize the relative error in Eq. (4). Then we evaluate the relative error between $f_{\text {out }}$ to $v(t)$ as the performance measure over 1000 discrete sampled time steps for $t \in(5000,6000)$. Through the three tasks, we again use $N=100$ oscillators and the uniform distribution in $[1,1.3]$ for their natural frequency $w_{i}$.

Fig. 3 depicts the errors in three tasks with respect the parameters $K$ and $\rho$. One can see in each task that minimum error occurs along a diagonal line. The line forms a clear border across which the error jumps from the low error regime (red) to high error regime (yellow). It should be noted that the three lines are identical and the same as the aligned critical points in Figure 2.(a) where the explosive death of the nodes occurs. This assures that the computational performance of the reservoirs is maximized near the first order phase transition.

As a final remark, it should be pointed out that the reservoir in Eq. (2) provides decent performance as a learning algorithm compared to other types of RC, for example, an echo state network(ESN). We applied a standard ESN in Lukoševičius et al. [26] to the above three tasks for comparison. The parameters $\rho$ and $K$ in Eq. (2) are chosen on the diagonal line in Fig. 3 so that the reservoir enjoys a criticality at the border between two phases. It turned out that the reservoir in Eq. (2) shows competitive(Task 1) or better(Task 2 and 3) results as compared to a ESN with 1000 nodes.

\subsection{Information capacity of regular and chaotic reservoirs}

In the previous work [7], a reservoir that consists of regular phase oscillators was presented as

$\theta_{i}^{\prime}=\omega_{i}+\frac{\lambda\left|w_{i}\right|}{k_{i}} \sum_{j=1}^{N} A_{i j} \sin \left(\theta_{j}-\theta_{i}\right), \quad i=1, \ldots, N$

where $\lambda$ is the coupling strength of oscillators and $A_{i j}$ is the entry of the adjacency matrix of the network. Here $A_{i j}=1$ if $i \neq j$, otherwise 0 . The model in Eq. (11) is known to have a simultaneous synchronization at a certain coupling value $\lambda$ [38]. Being used as a reservoir, it shows great performance improvement across such critical point.

This section investigates how chaos enhances a criticality in RC. We compare the performance of the forementioned two critical reservoirs, chaotic one in Eq. (2) and regular one in Eq. (11), when both are being poised at the first order phase transition. From here on, we call the former $\mathrm{QC}$ (quenched chaos) and the latter ES(explosive synchronization). When comparing the performance of these reservoirs, it is necessary to consider that the number of equations required to implement a single node is different: if they have the same number of nodes, the computational cost for the reservoir of Eq. (2) is greater than that of the reservoir of Eq. (11), roughly, by a factor of three.

Fig. 4 (a)-(c) depict the errors of QC and ES in task 1 to 3, respectively, according to the number of nodes used for the reservoirs. It is observed that the errors continuously decrease and reach the minimum at 150 nodes or less. In all three tasks, the error of QC is at least 1000 times smaller than that of ES. This indicates that QC excels by far ES, even when considering the forementioned difference in computation complexity between two reservoirs.

One of possible explanations on superiority of the chaotic reservoir is that the computing capability of critical reservoirs may depend on the collapsed dimension of attractors of reservoirs across the critical point. That is, the effect of criticality on computing performance may be related to how much reduction occurs in the dimension of the synchronization manifold at the phase transition. One can guess that the collapsed dimension of Eq. (2) at the explosive death is much greater than that of Eq. (11), from the fact that an attractor of a single Lorenz system has a greater Hausdorff dimension( $\sim 2.06$ ), compared to one dimensional attractor of a phase oscillator in Eq. (11). Computing the dimension of an attractor of a large coupled chaotic system is, however, extremely time-consuming and not practical. 
(a) Task 1

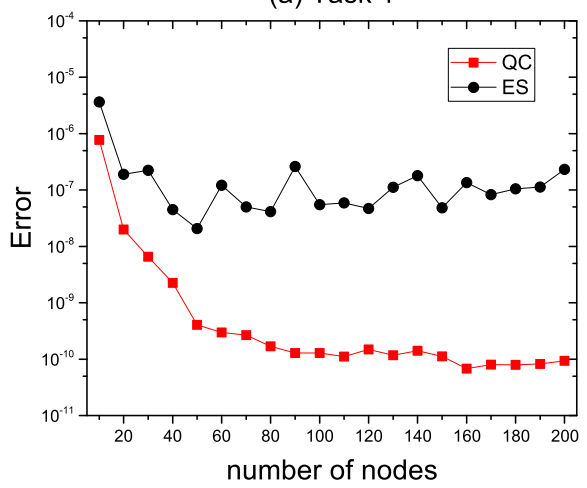

(b) Task 2

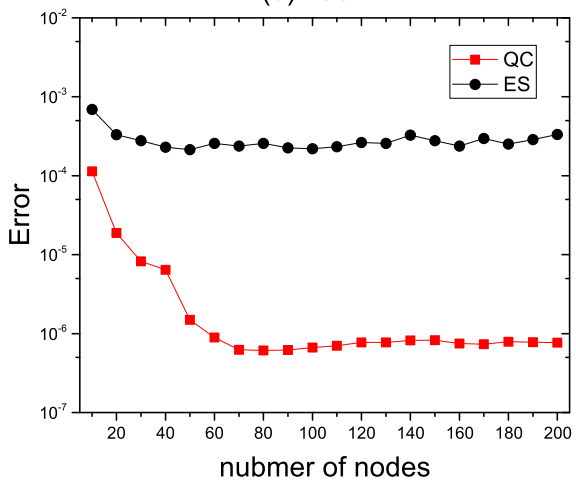

(c) Task 3

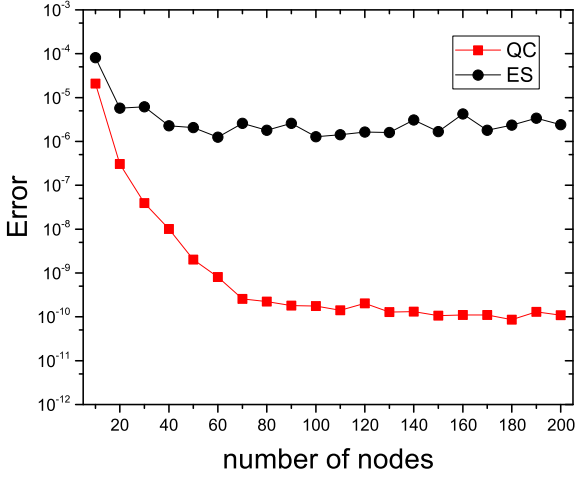

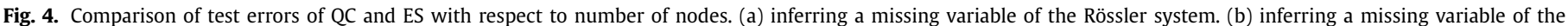
Chua's circuit. (c) filtering the Mackey-Glass equation .

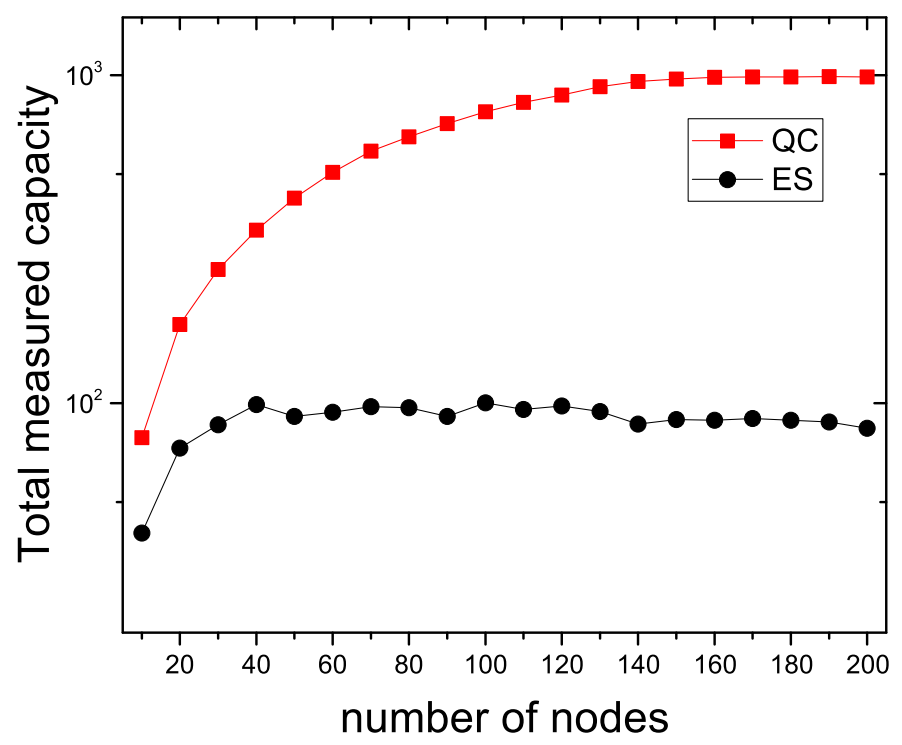

Fig. 5. Total information capacity of QC and ES with respect to number of oscillators. The most of the parameters for evaluation are from [9].

To overcome such difficulty in analyzing reservoir's internal structure, we rather adopt a measure that focuses on external functional capacity of systems. Here we use the total information capacity which is developed to compute the capacity of any input driven dynamical systems [9]. The total information capacity, roughly put, is defined as the assessment of reconstructing the set of orthonormal functions which is a basis of the fading memory Hilbert space. We refer the reader to [9] for more details.

Fig. 5 compares the total information capacity of QC and ES. We confirm that the capacity of QC continuously increases even near 150 nodes then decrease in tendency, while ES only increases till about 50 nodes, which agrees with the results of the numerical tasks in Fig. 4.

\section{Discussion}

In this work, we showed that the coupled chaotic systems can be used for efficient reservoir computing. The chaotic reservoirs can create a large criticality at the first order phase transition to create a ground state for computation. It notices in several computing tasks that the chaotic reservoirs excel the regular reservoirs, which is also confirmed from comparing their information capacity.
The results imply that using chaotic nodes is more beneficial in constructing reservoirs. This finding is important in several aspects. First of all, chaos is widely observed in neuronal systems, both experimentally and theoretically [1]. We confirmed that such ubiquity of chaos can be justified from the perspective of computing performance. That is, as long as it is properly quenched in the critical regime, chaos is an goal worth pursuing rather than an undesirable state to be avoided. Chaos computing is the paradigm that exploits the controlled richness of nonlinear dynamics to do flexible computations. This work shows another theoretical direction of chaos computing different from the approach using chaotic elements to emulate different logic gates [32,33]. Basic understanding of a role of criticality in regular and chaotic reservoirs can be expected to shed light on how information is processed in quenched coupled nonlinear systems, potentially leading to proposition of a broad range of reservoirs.

\section{Declaration of Competing Interest}

The authors declare that they have no known competing financial interests or personal relationships that could have appeared to influence the work reported in this paper.

\section{CRediT authorship contribution statement}

Jaesung Choi: Conceptualization, Methodology, Writing - original draft. Pilwon Kim: Formal analysis, Visualization, Validation, Writing - review \& editing.

\section{Acknowledgments}

This work was supported by the National Research Foundation of Korea (NRF-2017R1D1A1B04032921). The funder had no role in study design, data collection and analysis, decision to publish, or preparation of the manuscript.

\section{References}

[1] Aihara K. Chaotic oscillations and bifurcations in squid giant axons. Chaos 1986:257-69.

[2] Babloyantz A, Lourenco C. Computation with chaos: a paradigm for cortical activity. Proc Natl Acad Sci 1994;91(19):9027-31.

[3] Beggs JM, Plenz D. Neuronal avalanches in neocortical circuits. J Neurosci 2003;23(35):11167-77.

[4] Beggs JM, Timme N. Being critical of criticality in the brain. Front Physiol 2012;3:163.

[5] Botcharova M, Farmer SF, Berthouze L. Markers of criticality in phase synchronization. Front Syst Neurosci 2014;8:176.

[6] Casdagli M. Nonlinear prediction of chaotic time series. Phys D 1989;35(3):335-56.

[7] Choi J, Kim P. Critical neuromorphic computing based on explosive synchronization. Chaos 2019;29(4):043110. 
[8] Crowley MF, Epstein IR. Experimental and theoretical studies of a coupled chemical oscillator: phase death, multistability and in-phase and out-of-phase entrainment. J Phys Chem 1989;93(6):2496-502.

[9] Dambre J, Verstraeten D, Schrauwen B, Massar S. Information processing capacity of dynamical systems. Sci Rep 2012;2:514.

[10] Del Papa B, Priesemann V, Triesch J. Criticality meets learning: criticality signatures in a self-organizing recurrent neural network. PLoS One 2017; 12(5):e0178683.

[11] Dolnik M, Epstein IR. Coupled chaotic chemical oscillators. Phys Rev E 1996;54(4):3361.

[12] Du C, Cai F, Zidan MA, Ma W, Lee SH, Lu WD. Reservoir computing using dynamic memristors for temporal information processing. Nat Commun 2017;8(1):2204

[13] Ermentrout G, Kopell N. Oscillator death in systems of coupled neural oscillators. SIAM J Appl Math 1990;50(1):125-46.

[14] Goudarzi A, Teuscher C. Reservoir computing: quo vadis?. In: Proceedings of the 3rd ACM international conference on nanoscale computing and communication. ACM; 2016. p. 13.

[15] Herrero R, Figueras M, Rius J, Pi F, Orriols G. Experimental observation of the amplitude death effect in two coupled nonlinear oscillators. Phys Rev Lett 2000;84(23):5312.

[16] Jaeger H, Haas H. Harnessing nonlinearity: predicting chaotic systems and saving energy in wireless communication. Science 2004;304(5667):78-80.

[17] Jensen JH, Tufte G. Reservoir computing with a chaotic circuit. In: The 2019 conference on artificial life; 2017. p. 222-9. doi:10.1162/isal_a_039.

[18] Langton CG. Computation at the edge of chaos: phase transitions and emergent computation. Phys D 1990;42(1-3):12-37.

[19] Legenstein R, Maass W. Edge of chaos and prediction of computational performance for neural circuit models. Neural Netw 2007;20(3):323-34.

[20] Letellier C, Messager V. Influences on Otto E. Rössler's earliest paper on chaos. Int J Bifurc Chaos 2010;20(11):3585-616.

[21] Leyva I, Sevilla-Escoboza R, Buldú J, Sendina-Nadal I, Gómez-Gardeñes J, Arenas A, et al. Explosive first-order transition to synchrony in networked chaotic oscillators. Phys Rev Lett 2012;108(16):168702.

[22] Lourenço C. Attention-locked computation with chaotic neural nets. Int J Bifurc Chaos 2004;14(02):737-60.
[23] Lourenço C. Dynamical reservoir properties as network effects.. In: ESANN; 2006. p. 503-8.

[24] Lourenço C. Dynamical computation reservoir emerging within a biologica model network. Neurocomputing 2007;70(7-9):1177-85.

[25] Lourenço C. Structured reservoir computing with spatiotemporal chaotic attractors.. In: ESANN; 2007. p. 501-6.

[26] Lukoševičius M, Jaeger H, Schrauwen B. Reservoir computing trends. KI-Künstliche Intell 2012;26(4):365-71.

[27] Miller JF, Downing K. Evolution in materio: looking beyond the silicon box. In: Proceedings 2002 NASA/DoD conference on evolvable hardware. IEEE; 2002 p. $167-76$

28] Munoz MA. Colloquium: criticality and dynamical scaling in living systems. Rev Mod Phys 2018;90(3):031001.

[29] Ozden I, Venkataramani S, Long M, Connors B, Nurmikko A. Strong coupling of nonlinear electronic and biological oscillators: reaching the æamplitude deathg regime. Phys Rev Lett 2004;93(15):158102.

[30] Resmi V, Ambika G, Amritkar R, Rangarajan G. Amplitude death in complex networks induced by environment. Phys Rev E 2012;85(4):046211.

[31] Sharma A, Shrimali MD. Amplitude death with mean-field diffusion. Phys Rev E 2012;85(5):057204.

[32] Sinha S, Ditto WL. Dynamics based computation. Phys Rev Lett 1998;81(10):2156.

[33] Sinha S, Ditto WL. Computing with distributed chaos. Phys Rev E 1999;60(1):363.

[34] Tanaka G, Yamane T, Héroux JB, Nakane R, Kanazawa N, Takeda S, et al. Recent advances in physical reservoir computing: a review. Neural Netw 2019.

[35] Verma UK, Sharma A, Kamal NK, Kurths J, Shrimali MD. Explosive death induced by mean-field diffusion in identical oscillators. Sci Rep 2017:7(1):7936.

[36] Verma UK, Sharma A, Kamal NK, Shrimali MD. First order transition to oscillation death through an environment. Phys Lett A 2018;382(32):2122-6.

[37] Wei M-D, Lun J-C. Amplitude death in coupled chaotic solid-state lasers with cavity-configuration-dependent instabilities. Appl Phys Lett 2007;91(6):061121.

[38] Zhang X, Hu X, Kurths J, Liu Z. Explosive synchronization in a general complex network. Phys Rev E 2013;88(1):010802.

[39] Zhao N, Sun Z, Yang X, Xu W. Explosive death of conjugate coupled van der Pol oscillators on networks. Phys Rev E 2018;97(6):062203. 